\title{
Advantages of Ilizarov External Fixation in an Elderly Patient with Pilon Fracture with Severe Soft Tissue Injury and Severe Osteoporosis: A Case Report
}

\author{
Koji Nozaka*, Naohisa Miyakoshi, Hidetomo Saito, Shuichi Chida, Hiroyuki Tsuchie, \\ Yusuke Yuasa, Motoki Mita, Yoichi Shimada
}

Department of Orthopedic Surgery, Akita University Graduate School of Medicine, Hondo, Akita, Japan Email: ^kk-nozaka@mue.biglobe.ne.jp, miyakosh@doc.med.akita-u.ac.jp, hidetomo@doc.med.akita-u.ac.jp, shuichida@gmail.com, tuchikiti@yahoo.co.jp, yuasayusuke@med.akita-u.ac.jp,mmita@med.akita-u.ac.jp, seikei@doc.med.akita-u.ac.jp

How to cite this paper: Nozaka, K., Miyakoshi, N., Saito, H., Chida, S., Tsuchie, H., Yuasa, Y., Mita, M. and Shimada, Y. (2019) Advantages of Ilizarov External Fixation in an Elderly Patient with Pilon Fracture with Severe Soft Tissue Injury and Severe Osteoporosis: A Case Report. Open Journal of Orthopedics, 9, 14-22.

https://doi.org/10.4236/ojo.2019.91002

Received: November 25, 2018

Accepted: January 25, 2019

Published: January 28, 2019

Copyright $\odot 2019$ by author(s) and Scientific Research Publishing Inc. This work is licensed under the Creative Commons Attribution International License (CC BY 4.0).

http://creativecommons.org/licenses/by/4.0/

\begin{abstract}
Introduction: Pilon fracture in elderly individuals is characterized by senile skin atrophy, poor dermal extensibility, and thin subcutaneous tissue. The use of bulky internal fixation material can thus cause the swelling that accompanies the fracture to induce secondary injury to skin tissue. In addition, initiation of postoperative weight-bearing is delayed due to bone fragility and difficulties with partial weight-bearing, causing a tendency toward prolonged hospitalization. Mean duration of hospitalization after pilon fracture for elderly patients in our department was 79.2 days. Case Presentation: An 80 -year-old woman with pilon fracture with soft tissue injury and severe osteoporosis was transferred to our department. The fracture was treated using Ilizarov external fixation. Fourteen days postoperatively, walking with full weight-bearing was permitted. The hospital stay was 28 days. The external fixator of the ankle was removed 87 days postoperatively, at which time the patient was anatomically and functionally recovered and able to walk unaided. Conclusion: Ilizarov external fixation may represent a useful option in elderly patients with pilon fracture showing severe soft tissue injury and severe osteoporosis. The present case provides evidence that this procedure can be successfully applied to the management of such pilon fractures in elderly patients.
\end{abstract}

\section{Keywords}

Ilizarov External Fixation, Elderly Patient, Pilon Fracture, Soft Tissue Injury, Osteoporosis 


\section{Introduction}

Pilon fracture of the ankle in elderly individuals is characterized by senile skin atrophy, poor dermal extensibility, and thin subcutaneous tissue. The use of bulky internal fixation materials can thus cause the swelling that accompanies a fracture to induce secondary injury of the skin tissue. Due to bone fragility and difficulty in partial weight-bearing, elderly patients with periarticular fracture of the ankle experience delayed initiation of postoperative weight-bearing, prolonged hospitalization, and a tendency toward progression of disuse syndrome. The mean duration of hospitalization after pilon fracture in our department is 79.2 days. The length of hospital stay after pilon fracture in Japan is much longer than in Western countries, largely because there are extremely few nursing homes and rehabilitation hospitals in Japan [1]. Ilizarov external fixation for soft tissue injury and bones with osteoporosis, even in pilon fractures where the cortical bone is thin and use of screws for internal fixation is difficult, allows suitable fixation using multiple Ilizarov wires [2] [3]. Ilizarov external fixation is to prevent soft tissue complications by avoiding unnecessary internal fixation of fragile skin. The other is to enable early weight bearing, early ambulation, early independence, and early hospital discharge. We have been performing osteosynthesis using an Ilizarov external fixator and early weight-bearing in an elderly patient who sustained a pilon fracture with severe soft tissue injury and osteoporosis [4].

\section{Case Presentation}

An 80-year-old woman was injured when she fell on a snowy road. She was transported by ambulance to another hospital. Since comminuted fracture was observed, she was treated with skeletal traction. However, repositioning the fractured bone proved very difficult. Because of her low weight $(35 \mathrm{~kg})$, it was very difficult to apply skeletal traction with a heavy weight. Severe osteoporosis with cortical thinning at the fracture site was evident (Figure 1). Since skin condition was also not improving, she was transferred to our department, where left pilon fracture was diagnosed (OTA [Orthopaedic Trauma Association's Fracture and Dislocation Compendium] 43C3) (Figure 2). The bone mineral density of the lumbar spine (L2-4, $0.549 \mathrm{~g} / \mathrm{cm}^{2}$, T-score: $-3.78 \mathrm{~S}$.D.) and proximal femur $\left(0.622 \mathrm{~g} / \mathrm{cm}^{2}\right.$, T-score: -2.57 S.D. $)$ confirmed a diagnosis of osteoporosis. Skin necrosis was identified at the anterior fracture site (Figure 3). Given these findings, the risk of skin disorders with the use of bulky internal fixation materials appeared high. The decision was therefore made to use closed indirect reduction techniques with an Ilizarov ring fixator for rigid fixation [5]. One day after admission to our institute, Ilizarov ring fixator surgery was performed with the patient under general anesthesia in a supine position with trans-calcaneal traction. We used 4 rings for the Ilizarov fixator. The amount of reduction achieved by ligamentotaxis was checked using intensification. The foot was incorporated by olive wires inserted into the calcaneus and fixed to a foot ring connected to the 

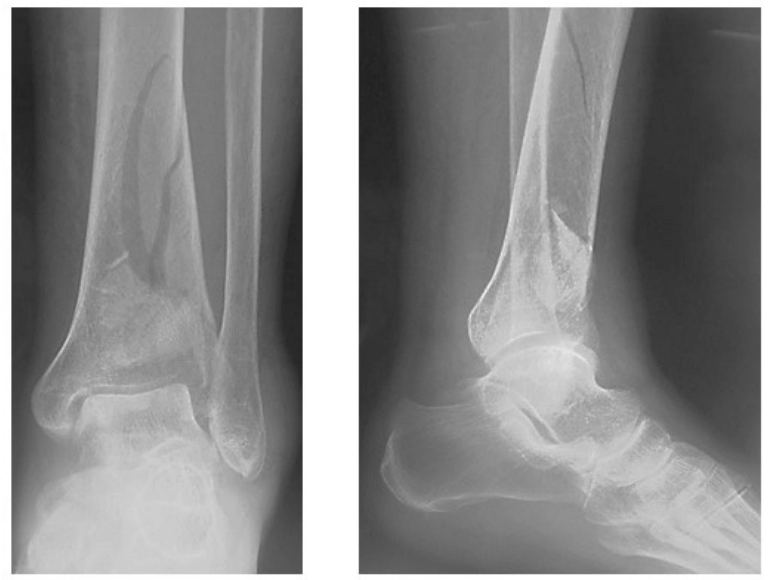

Figure 1. Preoperative radiographs on initial consultation showing pilon fracture.
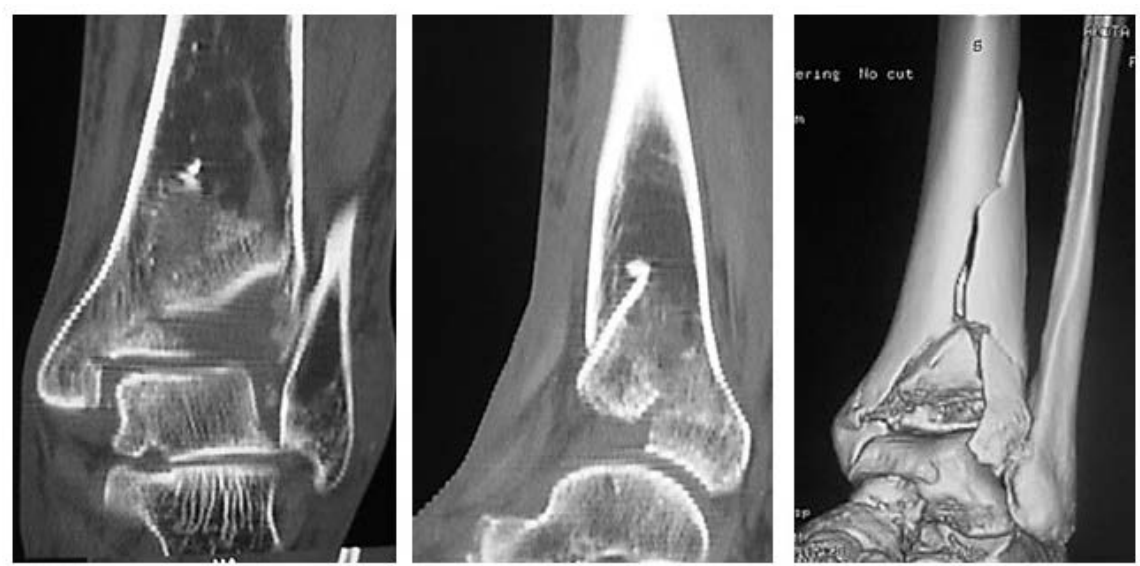

Figure 2. Computed tomography (CT) and 3-dimensional CT on initial consultation at our hospital show pilon fracture (OTA (Orthopaedic Trauma Association's Fracture and Dislocation Compendium) type 43C3).

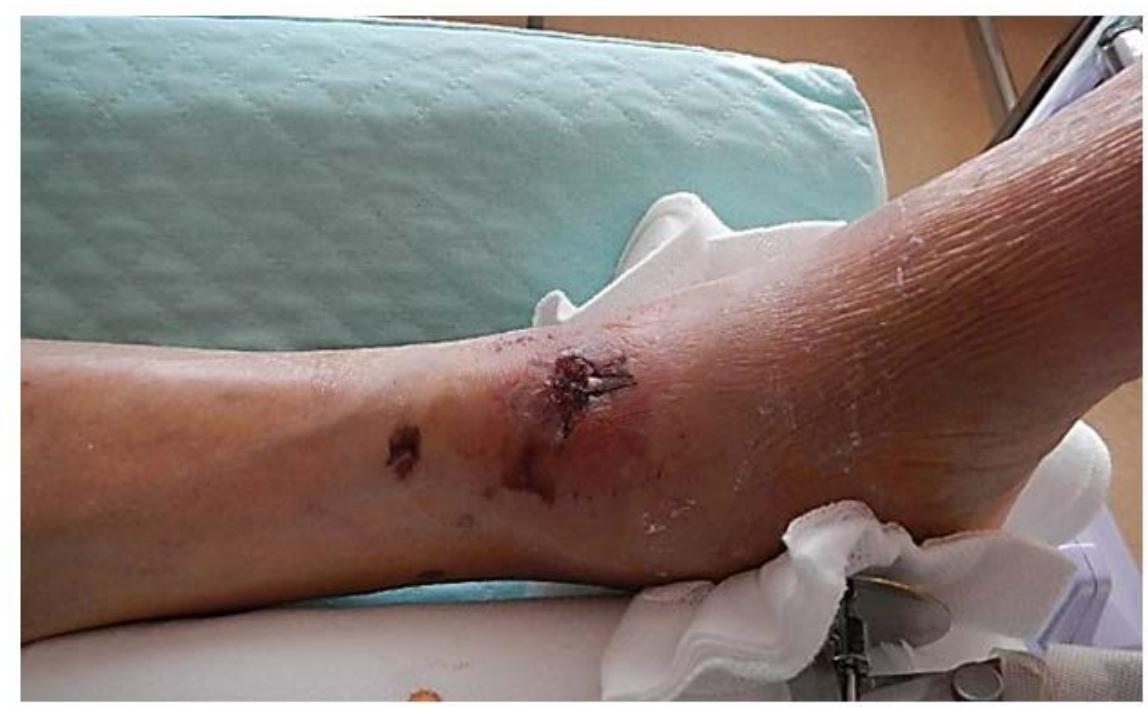

Figure 3. Skin condition at the time of transfer to our department. Necrosis is evident at the anterior fracture site. 
tibial external fixator (Figure 4). We placed traction on a foot ring while hanging varus, valgus, dorsiflexion and plantar flexion by ligamentotaxis in the distal direction [5]. Repositioning was gradually achieved, taking great care in accurate reduction of the intra-articular fragments; reduction was obtained with minimally invasive maneuvers performed with a smooth $3 \mathrm{~mm}$ wire used as a joystick and stabilized with a $1.8 \mathrm{~mm}$ wire. Alignment was continually checked by image intensification in both planes [6]. Distally migrated fragments were able to be replaced in the correct position with ligamentotaxis using fluoroscopic checks. An olive wire was also introduced through the tibial malleolus, first to reduce the fracture tilted in varus and anteriorly and then to compress the fracture site (Figure 3). After restoration of articular congruity, a $1.8 \mathrm{~mm}$ Ilizarov wire was passed parallel to the articular surface in the anteroposterior view on X-ray, approximately $15 \mathrm{~mm}$ from the joint line in the tibial epiphysis. Additional $1.8 \mathrm{~mm}$ Ilizarov wires were inserted in safe corridors to improve alignment and increase stability. Three wires were inserted onto the distal tibial ring. The wires were fixed to the rings of the fixator and tensioned. The foot was fixed in neutral position to avoid supination and equinus position. Olive wires were used in an opposing configuration, allowing variable degrees of interfragmentary compression to be achieved. This was of particular benefit in putting transverse compressive forces on a spiral metaphyseal fracture (Figure 4). We treated the patient with an ankle-hinge Ilizarov external fixator to allow early implementation of range-of-motion exercises (Figure 5) [7]. Fourteen days postoperatively, walking with full weight-bearing was permitted. The duration of hospitalization was 28 days. The external fixator of the ankle was removed at 87 days after surgery. Radiographs and computed tomography (CT) showed healing of the fracture at 87 days postoperatively (Figure 6 and Figure 7). At follow-up 3 years after surgery, the patient was satisfied with the procedure and was able to walk unaided. Clinical outcomes were measured using postoperative American Orthopaedic Foot \& Ankle Society scale ankle/hindfoot scale (AOFAS) score, Short Form-36

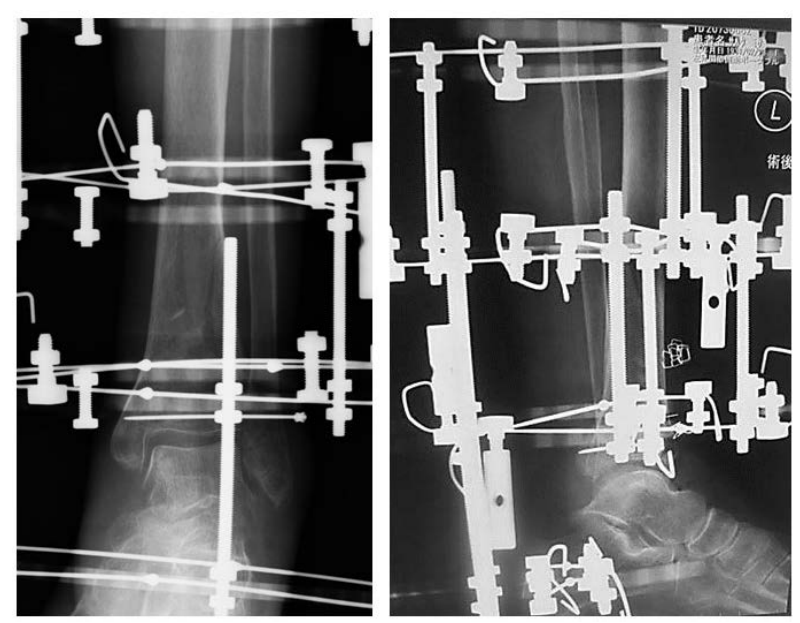

Figure 4. Anteroposterior- and lateral-view plain radiograph after surgery, showing anatomical reduction of the articular surface. 


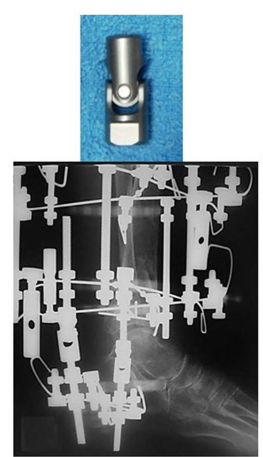

(a)

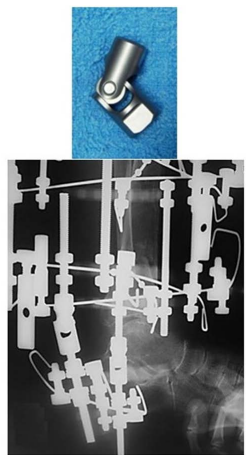

(b)

Figure 5. Ankle-hinge Ilizarov external fixation. (a) Neutral position; (b) Extended position.
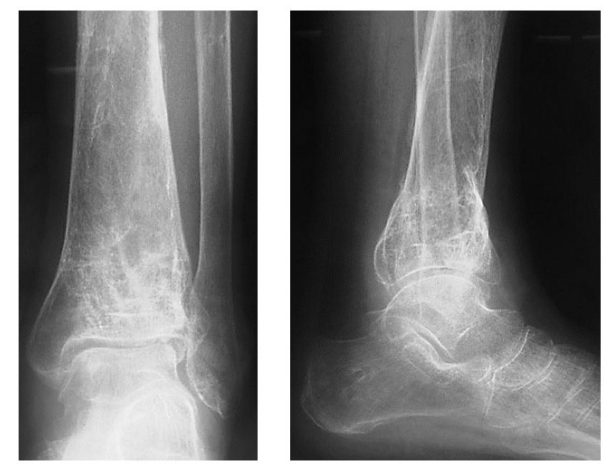

Figure 6. Radiograph after removal of the external fixator, showing bone union.
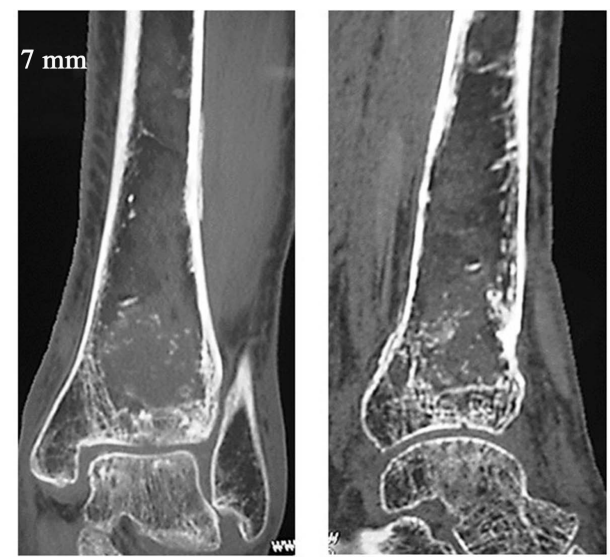

Figure 7. Anteroposterior- and lateral-view CT after removal of the external fixator, showing anatomical reduction of the articular surface.

(SF-36), and Visual Analogue Scale (VAS) pain questionnaires. AOFAS score was 97 after surgery. Postoperative SF-36 subscores were 40.2 for physical component summary after surgery, and 60.9 for mental component summary. Visual analogue scale score was 0 after surgery. As of the last visit, the degree of dorsal/plantar flexion was $0^{\circ} / 35^{\circ}$, range of motion of the ankle on the operated ankle had returned to almost matching the range of motion in the unoperated ankle (Figure 8). 


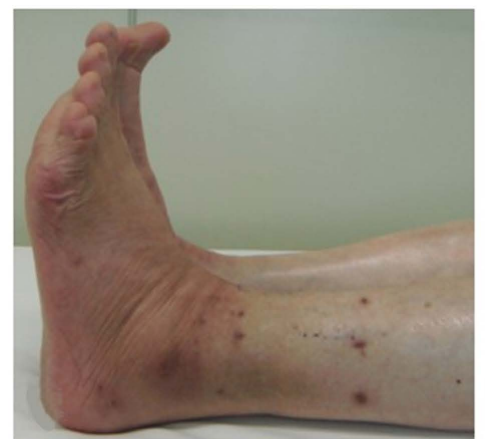

(a)

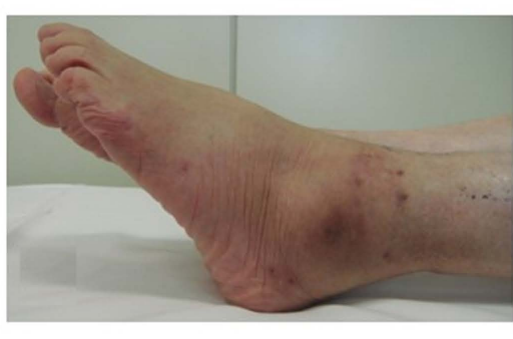

(b)

Figure 8. Final function of the ankle. (a) Extended position; (b) Flexed position.

\section{Discussion}

OTA type $\mathrm{C}$ pilon fractures are among the most difficult orthopedic trauma injuries to treat in the elderly [8]. Pilon fracture is characterized by thinning of the subcutaneous tissue, poor dermal extensibility, and, particularly in elderly individuals, senile skin atrophy [9]. Cutaneous aging manifests as a progressive reduction in maximum function and reserve capacity of skin tissue. Collagen atrophy is a major factor in skin aging, which is associated with a progressive increase in extensibility and reduced elasticity. With increasing age, the skin also becomes more fragile and susceptible to trauma, leading to more lacerations and bruising [10]. Furthermore, wound healing is impaired in the elderly. In recent years, lower profile metallic implants have significantly reduced soft-tissue complications, but cases of implant-related soft-tissue problems are still encountered [8]. For these reasons, treatment of type $\mathrm{C}$ pilon fracture remains challenging and controversial. External fixation techniques preserve soft tissues and the periosteum, yet provide stable reduction for OTA type C pilon fractures. Soft-tissue complications and deep infections are infrequent when external fixation is combined with minimally invasive surgery, with a frequency of $5 \%$ reported by Wyrsch, 1 of 17 cases by Tornetta, and 3 of 37 cases by Barbieri. Lovisetti et al. reported no cases of pseudoarthrosis or deep infection [6] [11] [12]. They attributed the $100 \%$ union rate to meticulous preservation of soft tissues in the fracture zone, made possible by the treatment strategy. Despite the quality of reduction achieved and lack of complications observed, clinical outcomes in their series have been less favorable than described by others. Treatment of pilon fractures by circular external fixation allows for less soft tissue dissection and represents a reliable method for achieving stabilization and healing of distal tibial intra-articular fractures with fewer soft tissue complications in the elderly.

Use of the Ilizarov external fixator is a safe method for pilon fracture in the elderly, with the advantage of immediate mobilization at full weight-bearing. The ankle-hinged Ilizarov external fixator and early joint movement allows effective management of tibial pilon fractures, usually accompanied by soft-tissue injuries [7]. Many reports have emphasized the negative impacts of non-weightbearing, including an approximately 50\% reduction in tibial cancellous bone 
mass in just 1 week, and the necessity for weight-bearing lasting at least twice as long for bone mass recovery even once weight-bearing has been restarted [13] [14] [15] [16]. The drop in physical fitness with aging is an unavoidable fact. Crutches require patients to maintain balance while weight is shifted in a swinging motion to move forward. Crutches are unstable for the elderly and represent a potential for further injury. Otherwise, body strength and bone strength deteriorate rapidly when the patient is confined to a wheelchair. Buildings and houses are very small in Japan, compared to the United States. This means it is difficult for a patient to move in a room with a Roll-A-Bout [17]. We do not have the Roll-A-Bout in Japan. Rehabilitation function is very weak at hospitals in Japan. Numbers of staff and nurses at hospitals in Japan are very low. In the present case, the patient made satisfactory progress to the point where discharge was possible at 28 days postoperatively. Rigid fixation and closed reduction of the Ilizarov external fixation enabled early weight-bearing and early discharge in the present case with severe soft tissue injury and severe osteoporosis.

\section{Conclusion}

In conclusion, Ilizarov external fixation for soft-tissue injury and bones with osteoporosis, even in pilon fractures where the cortical bone is thin and the use of screws for internal fixation is difficult, allows suitable fixation and closed reduction to be achieved using multiple Ilizarov wires. We have been performing osteosynthesis using an Ilizarov external fixator and early weight-bearing in elderly patients with pilon fracture with severe soft-tissue injury and osteoporosis.

\section{Acknowledgements}

There is no substantial direct or indirect commercial financial incentive associated with publishing this article.

\section{Consent}

Written informed consent was obtained from the patient for publication of this Case Report and any accompanying images. A copy of the written consent is available for review by the Editor-in-Chief of this journal.

\section{Conflicts of Interest}

The authors declare that they have no competing interests. There is no substantial direct or indirect commercial financial incentive associated with publishing this article.

Each author certifies that he or she has no commercial associations (e.g., consultancies, stock ownership, equity interest, patent/licensing arrangements, etc.) that might pose a conflict of interest in connection with the submitted article.

\section{Ethical Review Committee Statement}

It does not need to approve for a case report at Medical Ethics Review Committee 
in Akita University Graduate School of Medicine.

\section{References}

[1] Terada, S., Oshima, E., Yokota, O., Ikeda, C., Nagao, S., Takeda, N., Sasaki, K. and Uchitomi, Y. (2013) Person-Centered Care and Quality of Life of Patients with Dementia in Long-Term Care Facilities. Psychiatry Research, 205, 103-108. https://doi.org/10.1016/j.psychres.2012.08.028

[2] Faroug, R., Stirling, P. and Ali, F. (2013) A Novel Technique for Closed Reduction and Fixation of Paediatric Calcaneal Fracture Dislocation Injuries. Case Reports in Orthopedics, 2013, Article ID: 653146.

[3] Cavusoglu, A.T., Ozsoy, M.H., Dincel, V.E., Sakaogullari, A., Basarir, K. and Ugurlu, M. (2009) The Use of a Low-Profile Ilizarov External Fixator in the Treatment of Complex Fractures and Non-Unions of the Distal Femur. Acta Orthopaedica Belgica, 75, 209-218.

[4] Black, J.D., Bhavikatti, M., Al-Hadithy, N., Hakmi, A. and Kitson, J. (2013) Early Weight-Bearing in Operatively Fixed Ankle Fractures: A Systematic Review. The Foot, 23, 78-85. https://doi.org/10.1016/j.foot.2013.05.002

[5] Bozkurt, M., Ocguder, D.A., Ugurlu, M. and Kalkan, T. (2008) Tibial Pilon Fracture Repair Using Ilizarov External Fixation, Capsuloligamentotaxis, and Early Rehabilitation of the Ankle. The Journal of Foot and Ankle Surgery, 47, 302-306. https://doi.org/10.1053/j.jfas.2008.02.013

[6] Lovisetti, G., Agus, M.A., Pace, F., Capitani, D. and Sala, F. (2009) Management of Distal Tibial Intra-Articular Fractures with Circular External Fixation. Strategies in Trauma and Limb Reconstruction, 4, 1-6. https://doi.org/10.1007/s11751-009-0050-7

[7] Fırat, A., Tecimel, O., Işık, C., Ozdemir, M., Oçgüder, A. and Bozkurt, M. (2013) Ilizarov External Fixator in the Management of Tibial Pilon Fractures: Ankle Hinged vs Ankle Fixed Frame. Eklem Hastalıkları ve Cerrahisi, 24, 133-138. https://doi.org/10.5606/ehc.2013.30

[8] Bacon, S., Smith, W.R., Morgan, S.J., Hasenboehler, E., Philips, G., Williams, A., Ziran, B.H. and Stahel, P.F. (2008) A Retrospective Analysis of Comminuted Intra-Articular Fractures of the Tibial Plafond: Open Reduction and Internal Fixation versus External Ilizarov Fixation. Injury, 39, 196-202.

https://doi.org/10.1016/j.injury.2007.09.003

[9] Crist, B.D., Khazzam, M., Murtha, Y.M. and Della Rocca, G.J. (2011) Pilon Fractures: Advances in Surgical Management. Journal of the American Academy of Orthopaedic Surgeons, 19, 612-622. https://doi.org/10.5435/00124635-201110000-00005

[10] Calleja-Agius, J., Muscat-Baron, Y. and Brincat, M.P. (2007) Skin Ageing. Menopause International, 13, 60-64. https://doi.org/10.1258/175404507780796325

[11] Wyrsch, B., McFerran, M.A., McAndrew, M., Limbird, T.J., Harper, M.C., Johnson, K.D. and Schwartz, H.S. (1996) Operative Treatment of Fractures of the Tibial Plafond. A Randomized Prospective Study. The Journal of Bone and Joint Surgery, 78, 1646-1657. https://doi.org/10.2106/00004623-199611000-00003

[12] Tornetta, P., Weinwer, L., Bergman, M., Watnik, N., Steuer, J., Kelley, M. and Yang, E. (1993) Pilon Fractures: Treatment with Combined Internal and External Fixation. Journal of Orthopaedic Trauma, 7, 489-496. https://doi.org/10.1097/00005131-199312000-00001

[13] Sakai, A., Nakamura, T., Tsurukami, H., Okazaki, R., Nishida, S., Tanaka, Y., No- 
rimura, T. and Suzuki, K. (1996) Bone Marrow Capacity for Bone Cells and Trabecular Bone Turnover in Immobilized Tibia after Sciatic Neurectomy in Mice. Bone, 18, 479-486. https://doi.org/10.1016/8756-3282(96)00042-7

[14] Sakai, A., Sakata, T., Ikeda, S., Uchida, S., Okazaki, R., Norimura, T., Hori, M. and Nakamura, T. (1999) Intermittent Administration of Human Parathyroid Hormone (1-34) Prevents Immobilization-Related Bone Loss by Regulating Bone Marrow Capacity for Bone Cells in ddY Mice. Journal of Bone and Mineral Research, 14, 1691-1699. https://doi.org/10.1359/jbmr.1999.14.10.1691

[15] Sakata, T., Sakai, A., Tsurukami, H., Okimoto, N., Okazaki, Y., Ikeda, S., Norimura, T. and Nakamura, T. (1999) Trabecular Bone Turnover and Bone Marrow Cell Development in Tail-Suspended Mice. Journal of Bone and Mineral Research, 14, 1596-1604. https://doi.org/10.1359/jbmr.1999.14.9.1596

[16] Sakai, A. and Nakamura, T. (2001) Changes in Trabecular Bone Turnover and Bone Marrow Cell Development in Tail-Suspended Mice. Journal of Musculoskeletal \& Neuronal Interactions, 14, 387-392.

[17] Goldman, S.M. and Chang, T. (1999) Roll-A-Bout: Complete Off-Loading Made Easy. The Journal of Foot and Ankle Surgery, 38, 427.

https://doi.org/10.1016/S1067-2516(99)80045-3 